\title{
OPEN Broadband Microwave Absorption by Logarithmic Spiral Metasurface
}

\begin{abstract}
Shubo Wang ${ }^{1}{ }^{1}$, Bo Hou ${ }^{2,3}$ \& Che Ting Chan ${ }^{4}$
Metamaterials have enabled the design of electromagnetic wave absorbers with unprecedented performance. Conventional metamaterial absorbers usually employ multiple structure components in one unit cell to achieve broadband absorption. Here, a simple metasurface microwave absorber is proposed that has one metal-backed logarithmic spiral resonator as the unit cell. It can absorb $>95 \%$ of normally incident microwave energy within the frequency range of $6 \mathrm{GHz}-37 \mathrm{GHz}$ as a result of the scale invariant geometry and the Fabry-Perot-type resonances of the resonator. The thickness of the metasurface is $5 \mathrm{~mm}$ and approaches the Rozanov limit of an optimal absorber. The physics underlying the broadband absorption is discussed. A comparison with Archimedean spiral metasurface is conducted to uncover the crucial role of scale invariance. The study opens a new direction of electromagnetic wave absorption by employing the scale invariance of Maxwell equations and may also be applied to the absorption of other classical waves such as sound.
\end{abstract}

The absorption of electromagnetic waves has continuously drawn attention due to various applications such as stealth, reduction in radiation exposure, and solar energy related technologies. Since the advent of metamaterials $^{1-3}$, a large number of studies have been carried out to explore the possibility of absorbing electromagnetic waves by using metamaterials ${ }^{4-23}$. In particular, several designs of metamaterials for achieving broadband absorption have been proposed, such as the dispersion-engineered metamaterial ${ }^{24,25}$, the pyramid metamaterial ${ }^{26,27}$, and the metasurface Salisbury screen ${ }^{28}$. While these designs are geometrically quite different, they all involve the assembling and fine tuning of multiple structural components in one unit cell in order to induce multiple electromagnetic resonances to broaden the absorption bandwidth. A simple design recipe of absorbers that can achieve broadband strong absorption of electromagnetic waves would be highly desirable. We will show that the scale invariance of Maxwell equations provides a possible solution to this problem. Following the recipe, we designed a metasurface consisting of logarithmic spiral resonators (LSRs) which can absorb $>95 \%$ of incident microwave within the frequency range of $6 \mathrm{GHz}-37 \mathrm{GHz}$. In contrast, a metasurface formed of Archimedean spiral resonators (ASRs), which has no scale invariant feature, only absorbs microwave strongly at particular frequencies. Note that several metamaterials consisting of spiral structures have been proposed for electromagnetic wave absorption $^{29-33}$, but their performance is narrow-banded.

Consider the source-free Maxwell equations in vacuum: $\nabla^{2} \mathbf{E}(\mathbf{r}, t)=\left(1 / c^{2}\right) \partial^{2} \mathbf{E}(\mathbf{r}, t) / \partial t^{2}, \nabla^{2} \mathbf{B}(\mathbf{r}, t)=$ $\left(1 / c^{2}\right) \partial^{2} \mathbf{B}(\mathbf{r}, t) / \partial t^{2}$. These equations are invariant under the transformation of $\mathbf{r} \rightarrow \chi \mathbf{r}$ and $t \rightarrow \chi t$, where $\chi$ is an arbitrary scaling factor. The scale invariance indicates that the electromagnetic response of a system remains unchanged under the scaling of both geometric dimensions and wavelength. In materials with weak dispersion, this invariance can be approximately maintained. Under this condition, it is possible to design a metamaterial with broadband properties (e.g. absorption) deriving from the scale invariance of its unit cell geometry. In contrast, the broadband properties of conventional metamaterials usually derive from multiple structures with different geometric dimensions, which induce electromagnetic resonances at different frequencies. We will show that metamaterials with a scale invariant unit cell geometry can kill two birds with one stone: they can not only achieve broadband absorption of electromagnetic waves, but also maintain a small thickness as the result of a space coiling feature.

\footnotetext{
${ }^{1}$ Department of Physics, City University of Hong Kong, Tat Chee Avenue, Kowloon, Hong Kong SAR, China. ${ }^{2}$ School of Physical Science and Technology \& Collaborative Innovation Center of Suzhou Nano Science and Technology, Soochow University, Suzhou, 215006, China. ${ }^{3}$ Key Laboratory of Modern Optical Technologies of Ministry of Education \& Key Lab of Advanced Optical Manufacturing Technologies of Jiangsu Province, Suzhou, 215006, China. ${ }^{4}$ Department of Physics, The Hong Kong University of Science and Technology, Clear Water Bay, Kowloon, Hong Kong SAR, China. Correspondence and requests for materials should be addressed to S.W. (email: shubwang@cityu. edu.hk) or C.T.C. (email: phchan@ust.hk)
} 
a

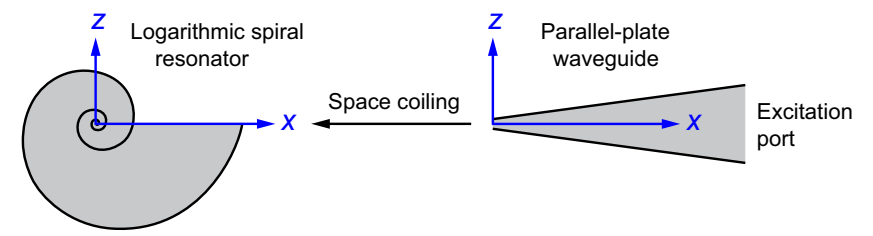

b

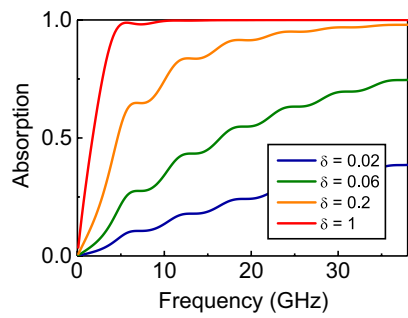

C

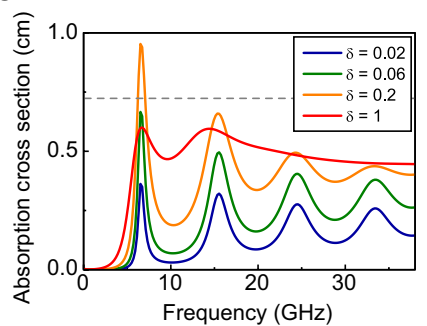

d

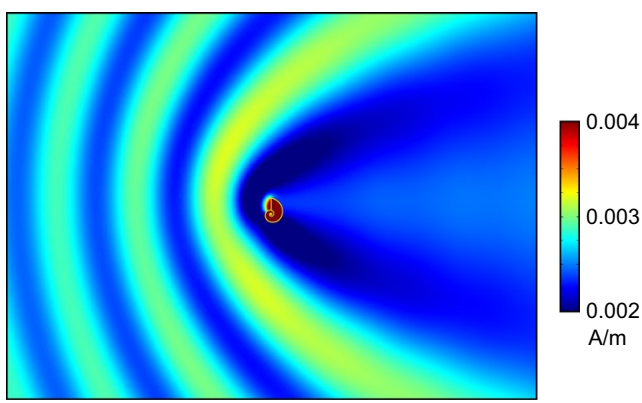

Figure 1. Absorption of single LSR. (a) The proposed LSR (left) is drawn to scale. It can be viewed as the coiling of a tapered parallel-plate waveguide (right). We set $\alpha=0.0031 \mathrm{~mm}, \beta=0.22$, and $\theta \in[0,33]$ (see text for the definition). (b) Absorption spectrum of the tapered parallel-plate waveguide for the fundamental TM mode under different loss of dielectric material $\left(\varepsilon_{\mathrm{r}}=1.4+i \delta\right)$. The width of the openings is $0.01 \mathrm{~mm}$ and $3.4 \mathrm{~mm}$. The total length is $21 \mathrm{~mm}$. The waveguide is excited from the right port. (c) Normalized absorption cross section of the LSR for different loss parameter $\delta$. (d) Magnetic field amplitude at $f=6.7 \mathrm{GHz}$ with $\delta=0.2$.

\section{Results}

Logarithmic spiral resonator. We consider the two-dimensional metamaterial unit shown in Fig. 1a. The geometry of the unit (left of Fig. 1a) is a logarithmic spiral defined by the function $r(\theta)=\alpha e^{\beta \theta}$, where $r, \theta$ are the polar coordinates and $\alpha, \beta$ are two arbitrary constants. An isotropic scaling of the spiral by a factor of $\chi$ leads to the transformation of $r \rightarrow \chi r=\chi \alpha e^{\beta \theta}=\alpha e^{\beta(\theta+\ln \chi / \beta)}$, which is identical to the rotation of $r(\theta)$ by an angle of $\ln \chi / \beta$. Therefore, an arbitrary scaling operation on the logarithmic spiral followed by a rotation recovers the original geometry, i.e., it is scale invariant. We consider a logarithmic spiral made of copper with conductivity $\sigma=5.96 \times 10^{6} \mathrm{~S} / \mathrm{m}$, filled with a dielectric material with relative permittivity $\varepsilon_{\mathrm{r}}=1.4+i \delta$ (grey region). The copper film has a thickness of $0.1 \mathrm{~mm}$. The spiral can be viewed as transformed from a tapered parallel-plate (TPP) waveguide through space coiling. Under TM polarization, i.e. magnetic field is polarized along $y$ direction in Fig. 1a, the TPP waveguide has a fundamental mode that has no cutoff frequency, which is similar to the normal parallel-plate waveguide. Figure $1 \mathrm{~b}$ shows the absorption of this mode in the TPP waveguide calculated by using COMSOL ${ }^{33}$ with port boundary condition. The absorption is defined as $1-\left|S_{11}\right|^{2}-\left|S_{21}\right|^{2}$, where $S_{11}$ and $S_{21}$ are the $S$-parameters. The length of the waveguide along $x$ direction is $\sim 21 \mathrm{~mm}$. The width of the left and the right openings is $0.01 \mathrm{~mm}$ and $3.4 \mathrm{~mm}$, respectively. We consider port excitation at the right opening. The variation of the waveguide's width induces impedance mismatch and hence Fabry-Perot-type resonances, which accounts for the peaks in the absorption spectrum in Fig. 1b. We notice that when the dielectric loss $\delta$ is large, the absorption can approach $100 \%$ in a wideband. However, the TPP waveguide is bulky. In contrast, the logarithmic spiral is geometrically compact and its scale invariance should give rise to similar absorption property at different frequencies. Figure 1c shows the normalized absorption cross section (ACS) of the LSR with $\alpha=0.0031 \mathrm{~mm}$, $\beta=0.22$ and $\theta \in[0,33]$, which is calculated by using COMSOL with open boundary condition (realized by a perfectly matched layer). The normalized ACS is defined as $C_{\mathrm{abs}}=-1 /(2 I) \oint \operatorname{Re}\left(\mathbf{E} \times \mathbf{H}^{*}\right) \cdot \hat{n} d A$, where $I$ is the intensity of incident microwave, $\mathbf{E}$ and $\mathbf{H}$ are the total fields, and $\hat{n}$ is the outward unit normal vector of the closed surface on which the integral is evaluated. We note that when $\delta$ is small, multiple peaks appear in the ACS spectrum due to the Fabry-Perot-type resonances. The frequencies of the peaks are determined by the total electric length of the TPP waveguide. As $\delta$ is increased, the ACS grows and can beat the theoretical upper limit of single channel subwavelength resonance (i.e. $\lambda / 2 \pi^{34}$, marked by the dashed line) as in the case of $\delta=0.2$ at $f=6.7 \mathrm{GHz}$. The resonances lead to an increase of both ACS and scattering cross section (SCS). Consequently, the LSR has a large extinction cross section as shown in Fig. $1 \mathrm{~d}$ for the magnetic field amplitude at $f=6.7 \mathrm{GHz}$. When $\delta$ is large enough, we obtain a broadband large ACS due to the scale invariance of the LSR.

Logarithmic spiral metasurface. We now employ the strong absorption of the LSR to build a metasurface (i.e. thin-layer metamaterial) microwave absorber. This is achieved by arranging the LSRs into a one-dimensional array with period $a=7.83 \mathrm{~mm}$ on a copper substrate, as shown in Fig. 2a. The opening of the LSRs are facing to the direction of incoming waves. The overall thickness of the metasurface is $d=5 \mathrm{~mm}$. The grey region denotes lossy dielectric material with relative permittivity $\varepsilon_{\mathrm{r}}=1.4+i$. We calculated the absorption of a TM-polarized plane wave normally incident on the metasurface. In the numerical simulations, periodic boundary condition is applied in the $x$ direction, and open boundary condition is applied in the $z$ direction. The results are shown in Fig. $2 \mathrm{~b}$ as the solid red line. We note that the metasurface can absorb $>95 \%$ of incident microwave energy within the frequency range of $7.7 \mathrm{GHz}-37 \mathrm{GHz}$. The lower bound here is determined by the lowest order resonance of the LSR. The upper bound is determined by the period of the metasurface. Above $37 \mathrm{GHz}$, the absorption reduces 
a

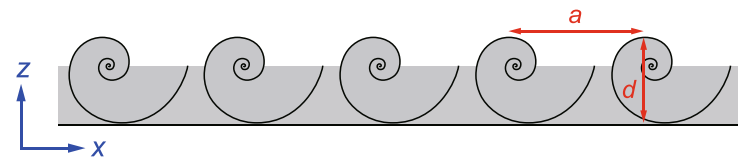

b
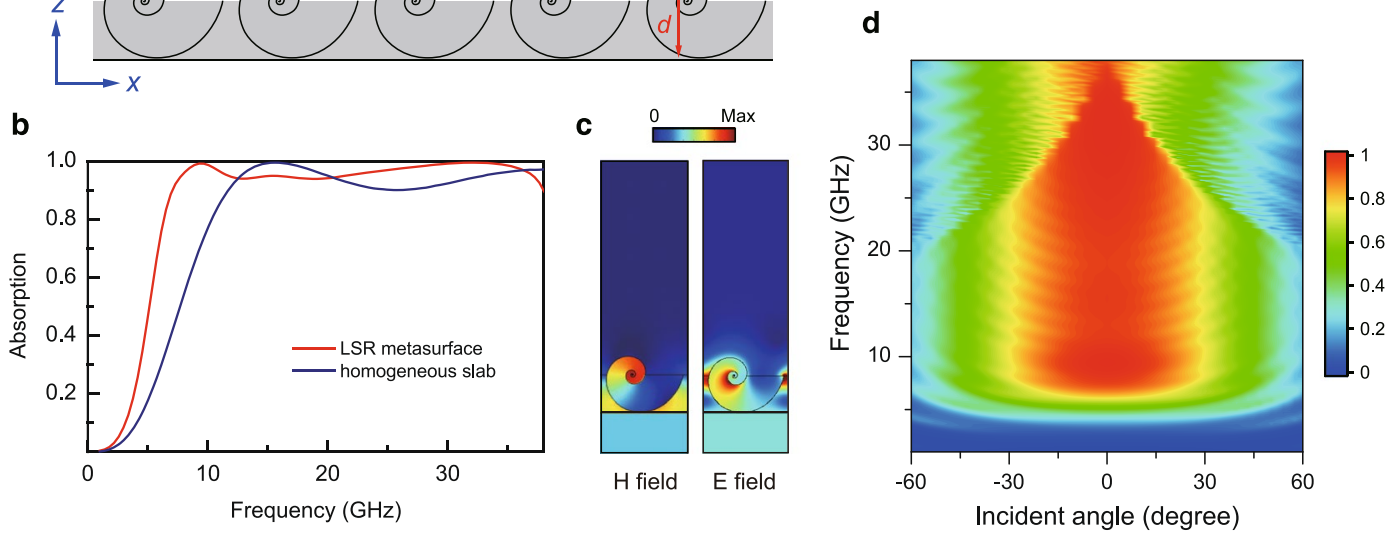

Figure 2. Absorption of the LSR metasurface. (a) The LSR metasurface with thickness $d=5 \mathrm{~mm}$ and period $a=7.83 \mathrm{~mm}$. The LSR is filled with dielectric material $\left(\varepsilon_{\mathrm{r}}=1.4+i\right)$. (b) Absorption of the LSR metasurface (red) and a homogeneous dielectric slab with $\varepsilon_{\mathrm{r}}=1.4+i$ (blue) under normal incidence of a TM-polarized plane wave. (c) Amplitude of the induced magnetic and electric fields at $f=10 \mathrm{GHz}$. (d) Absorption as a function of frequency and incident angle.

due to the effect of high-order diffractions. For comparison, we also calculated the absorption of a homogeneous slab with relative permittivity $\varepsilon_{\mathrm{r}}=1.4+i$, which is backed with a copper substrate. The results are shown in Fig. $2 \mathrm{~b}$ as the solid blue line. The lowest frequency with $95 \%$ absorption for the slab is $12.8 \mathrm{GHz}$ which is much higher than that of the LSR metasurface. Besides, the slab has a lower absorption of $\sim 90 \%$ at $20 \mathrm{GHz}-30 \mathrm{GHz}$. We note that high absorption at low frequencies is rather difficult to achieve due to the large wavelength relative to the thickness of the absorber. The LSR metasurface, therefore, enables the realization of thin microwave absorbers. The high absorption of the LSR metasurface is corroborated by the induced magnetic and electric fields shown in Fig. $2 \mathrm{c}$ for the case of $f=10 \mathrm{GHz}$. The fields are mainly concentrated in the center of the LSR due to the Fabry-Perot-type resonances and almost no field is reflected back. We then studied the dependence of the absorption on the incident angle of the microwave. Figure $2 \mathrm{~d}$ shows the numerically calculated absorption as a function of frequency and incident angle. We note that high absorption can be achieved for incident angles lying within $[-30,30]$ degrees. As the angle increases, the bandwidth of high absorption reduces. The dependence of absorption on the incident angle is attributed to the special symmetry of the LSR. We also note that the maximum absorption bandwidth appears in the case of normal incidence. In the cases of oblique incidence at high frequencies, high-order diffraction contributes to the reflected wave and causes a lower absorption.

If we optimize the LSR metasurface by using graded-index lossy dielectric as the filling material, we can further broaden its absorption bandwidth. Figure 3 a shows one unit cell of the optimized LSR metasurface which has a period of $a=7.45 \mathrm{~mm}$ and a thickness of $d=5 \mathrm{~mm}$. We set $\alpha=0.008 \mathrm{~mm}, \beta=0.2$, and $\theta \in[0,31.4]$. The grayscale indicates the absolute value of the permittivity. The first layer of the LSR has $\varepsilon_{\mathrm{r}}=1+\theta\left(\varepsilon_{c}-1\right) /(2 \pi)$ with $\varepsilon_{\mathrm{c}}=4.2+2.6 i$. The inner layers have $\varepsilon_{\mathrm{r}}=\varepsilon_{\mathrm{c}}$. The material outside the LSR is homogenous with $\varepsilon_{\mathrm{r}}=2+1.5 i$. For comparison, we also considered a slab absorber with graded permittivity $\varepsilon_{\mathrm{r}}=1+h\left(\varepsilon_{c}-1\right) / d$ as shown in Fig. 3b, where $d=5 \mathrm{~mm}$ and $h$ is the distance from the upper surface of the slab. We considered two cases with $\varepsilon_{\mathrm{c}}=4.2+2.6 i$ and $\varepsilon_{\mathrm{c}}=1.4+i$, respectively. The absorption spectra of the optimized structures are shown in Fig. 3c. The LSR metasurface can achieve $>95 \%$ absorption within $6 \mathrm{GHz}-37 \mathrm{GHz}$, which is much better than the two slab absorbers. We note that the theoretical limit on the thickness of a non-magnetic absorber can be determined with the Rozanov relation: $d \geq 1 /\left(2 \pi^{2}\right)\left|\int_{0}^{\infty} \ln \right| R(\lambda)|d \lambda|$, where $R$ is the reflection coefficient ${ }^{35}$. We found that the theoretical limit is about $4 \mathrm{~mm}$, which is $80 \%$ of the thickness of the proposed metasurface absorber.

The metasurface absorber in Fig. 2a is invariant along $y$ direction and mainly absorbs TM-polarized microwaves. To achieve high absorption for both TM and TE (i.e. electric field is along $y$ direction) polarizations, we then design a metasurface consisting of a two-dimensional array of LSRs on a copper surface. The unit cell is shown in Fig. 4a, where two identical LSRs are orthogonally arranged so that they can absorb both TM- and TE-polarized microwaves. Note that the LSRs are finite along axis direction with a thickness of $c=2.5 \mathrm{~mm}$. The unit cell has a dimension of $a \times b \times d=10.2 \mathrm{~mm} \times 7.2 \mathrm{~mm} \times 5.4 \mathrm{~mm}$. The dielectric material has a relative permittivity of $\varepsilon_{\mathrm{r}}=1.4+i$. We numerically calculated the absorption of the metasurface and the results are shown in Fig. 4b. In the numerical simulations, periodic boundary condition is applied in the $x$ and $y$ directions, and open boundary condition is applied in the $z$ direction. We note that for both $y$ (i.e. TM) and $x$ (i.e. TE) polarizations the absorption is above $90 \%$ within the frequency range of $8 \mathrm{GHz}-38 \mathrm{GHz}$. Compared to the previous case in Fig. 2b, the absorption is lower due to the truncation of the LSRs along the center axis direction, which leads to smaller quality factors of the resonances and hence a relatively weaker enhancement. Figure $4 \mathrm{c}, \mathrm{d}$ show the dependence of the absorption on the incident angle for both the $y$ and $x$ polarizations, respectively. In both cases, high absorption can be achieved for incident angle within $[-30,30]$ degrees. The different performances of the metasurface under $y$ and $x$ polarizations are due to the asymmetric orientation of the two LSRs in one unit cell. 
a b
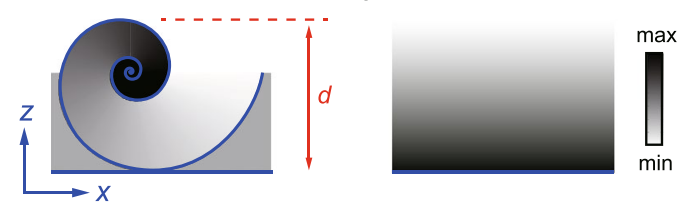

C

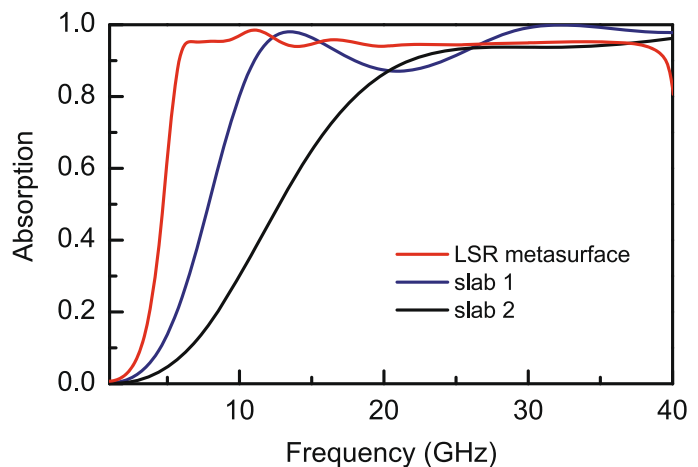

Figure 3. Absorption of the optimized LSR metasurface. (a) Unit cell of the optimized LSR metasurface. The first layer of the LSR is filled with a graded-index dielectric material with $\varepsilon_{\mathrm{r}}=1+\theta\left(\varepsilon_{\mathrm{c}}-1\right) /(2 \pi)$, where $\varepsilon_{\mathrm{c}}=4.2+2.6 i$. The inner layers have $\varepsilon_{\mathrm{r}}=\varepsilon_{\mathrm{c}}$. The dielectric outside the LSR has $\varepsilon_{\mathrm{r}}=2+1.5 i$. We set $\alpha=0.008 \mathrm{~mm}$, $\beta=0.2$, and $\theta \in[0,31.4]$. The unit cell has a period of $a=7.45 \mathrm{~mm}$ and a thickness of $d=5 \mathrm{~mm}$. The color indicates the magnitude of the permittivity. (b) A graded-index dielectric slab with $\varepsilon_{\mathrm{r}}=1+h\left(\varepsilon_{\mathrm{c}}-1\right) / d$, where $h$ is the distance from the upper surface of the slab. (c) Absorption of the optimized LSR metasurface (red), the gradedindex dielectric slab with $\varepsilon_{\mathrm{c}}=4.2+2.6 i$ (blue), and the graded-index dielectric slab with $\varepsilon_{\mathrm{c}}=1.4+i$ (black).

a
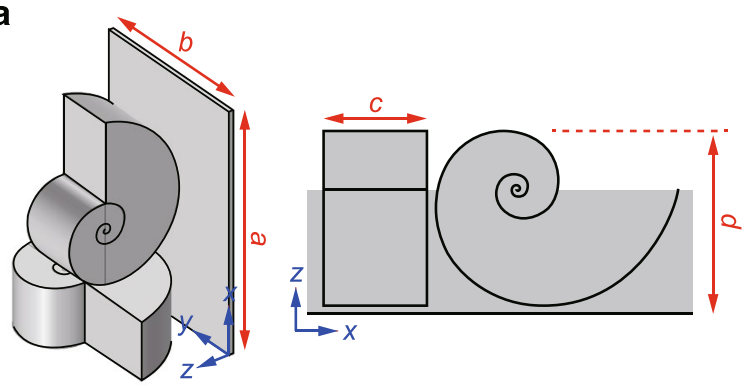
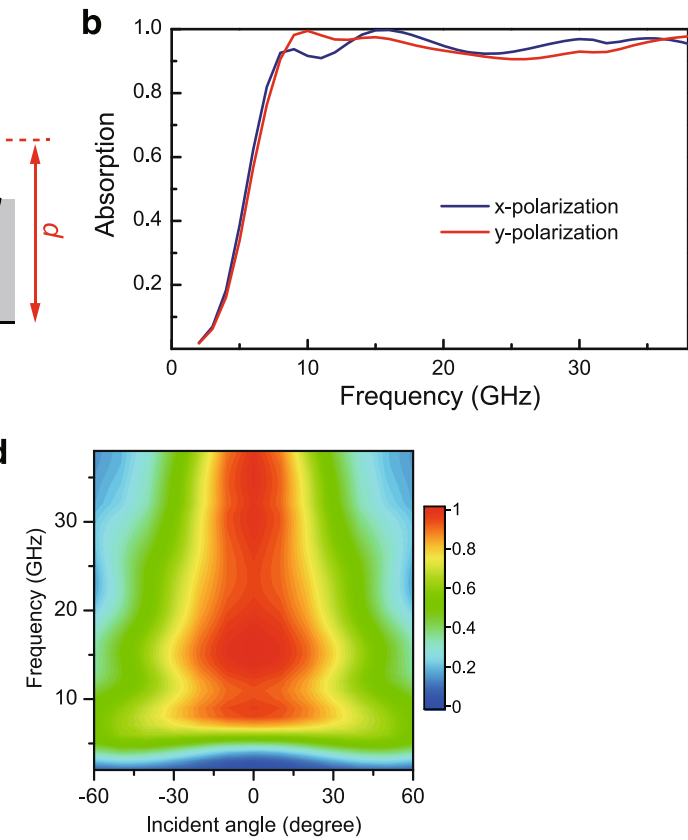

Figure 4. LSR metasurface for absorbing both TM- and TE-polarized microwaves. (a) Unit cell of the LSR metasurface. The two LSRs are orthogonal and backed with a metal surface. We set $a=10.2 \mathrm{~mm}, b=7.2 \mathrm{~mm}$, $c=2.5 \mathrm{~mm}$, and $d=5.4 \mathrm{~mm}$. (b) Absorption for incident wave polarized along $x$ and $y$ directions. (c) Angle dependence of the absorption for $y$ (i.e. TM) polarization. (d) Angle dependence of the absorption for $x$ (i.e. TE) polarization.

Archimedean spiral metasurface. To further understand the critical role of scale invariance in the broadband absorption, we performed a comparative study where the metasurface is constructed with ASRs. The ASR has a geometry that is not scale invariant, as shown by the inset in Fig. $5 \mathrm{a}$, which is defined by the function $r(\theta)=r_{0}+\rho \theta$ with $r_{0}$ and $\rho$ being constants. We set $r_{0}=0$ for simplicity. The ASR contains five layers with an 
a

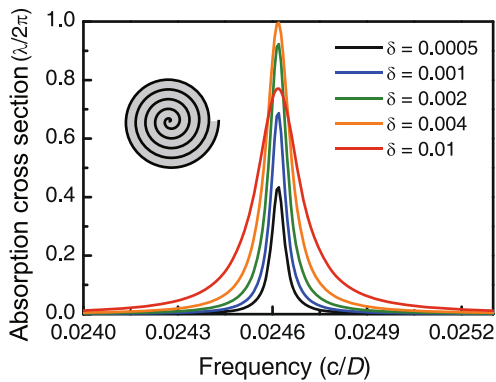

b

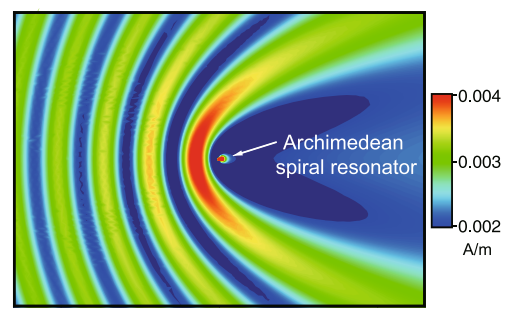

C

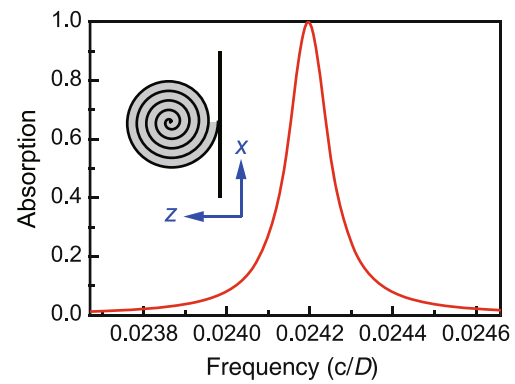

d

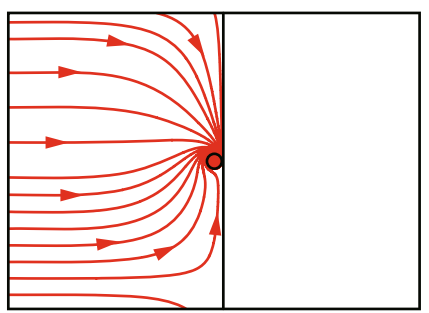

e

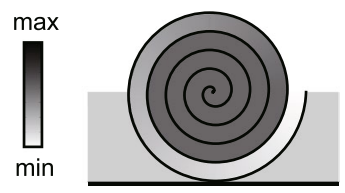

$\mathbf{f}$

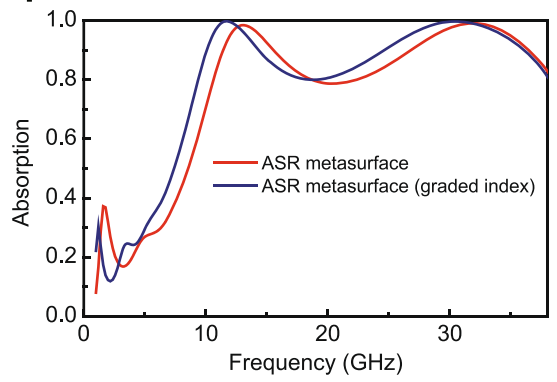

Figure 5. Absorption of single ASR and the ASR metasurface. (a) Absorption cross section of single ASR for dielectric materials with different loss parameters $\left(\varepsilon_{\mathrm{c}}=2+i \delta\right)$. The inset is a schematic of the ASR. (b) Magnetic field amplitude at the resonance frequency. The arrow marks the position of the ASR. (c) Absorption of the metasurface formed of ASRs backed with a metal surface. The metasurface has a period of $18 \mathrm{D}$ with $\mathrm{D}$ being the diameter of the ASR. (d) Poynting vectors in one unit cell of the metasurface, showing the singular behaviour of the ASR. (e) Unit cell of optimized ASR metasurface. The first layer is filled with a graded-index dielectric material with $\varepsilon_{\mathrm{r}}=1+\theta\left(\varepsilon_{\mathrm{c}}-1\right) /(2 \pi)$, where $\varepsilon_{\mathrm{c}}=4.2+2.6 i$. The inner layers have $\varepsilon_{\mathrm{r}}=\varepsilon_{\mathrm{c}}$. The dielectric outside the LSR has $\varepsilon_{\mathrm{r}}=2+1.5 i$. The thickness and period are the same as the LSR metasurface in Fig. 3a. (f) Absorption of the ASR metasurface with homogeneous dielectric of $\varepsilon_{\mathrm{r}}=1.4+i$ (red) and gradedindex dielectric (blue).

overall diameter of $D$ and is filled with dielectric material $\left(\varepsilon_{\mathrm{r}}=2+i \delta\right)$. Similar to the LSR, the ASR also supports Fabry-Perot-type resonances, which have been used to achieve unusual effective permeability in metamaterials ${ }^{1}$. The resonances can significantly enhance the ACS of the ASR, as shown in Fig. $5 \mathrm{a}$ for the enhancement by the lowest order resonance, which is calculated using COMSOL with open boundary condition. In contrast to the LSR, the large ACS of the ASR is narrowband. As the dielectric loss $\delta$ is increased, the ACS first grows and then decreases. The maximum absorption, i.e. the single channel theoretical upper limit $\lambda / 2 \pi$, is achieved at the normalized frequency $\sim f=0.0246 c / D$ in the case of $\delta=0.004$, where $c$ is the speed of light in vacuum. Figure 5b shows the magnetic field amplitude at the resonance frequency, where the ASR (marked by an arrow) induces a large extinction cross section. We then consider a metasurface absorber consisting of one-dimensional array of ASR and a reflection metal surface, as shown by the inset in Fig. $5 \mathrm{c}$. The period of the metasurface is $18 D$. The filling dielectric material has a relative permittivity of $\varepsilon_{\mathrm{r}}=2+0.005 i$. We calculated the absorption of the ASR metasurface and the results are shown in Fig. $5 c$ by the red solid line. In the numerical simulations, periodic boundary condition is applied in the $x$ direction, and open boundary condition is applied in the $z$ direction. Notice that near-perfect absorption can be achieved at the resonance frequency of $0.0242 c / D$. Figure $5 \mathrm{~d}$ shows the Poynting vectors within one unit cell at this frequency. We notice that the ASR behaves like an energy sink (i.e. singularity) that absorbs all the microwave in one unit cell. To compare the ASR metasurface with the LSR metasurface, we then set $\rho=0.09 \mathrm{~mm}$ so that the ASR has approximately the same volume as the LSR. We assume equal period and thickness for the two types of metasurfaces. Both homogeneous filling and graded-index filling are considered for comparison. Figure 5e shows the ASR metasurface with graded-index filling, where the outer layer has permittivity $\varepsilon_{\mathrm{r}}=1+\theta\left(\varepsilon_{\mathrm{c}}-1\right) /(2 \pi)$ with $\varepsilon_{\mathrm{c}}=4.2+2.6 i$ and the inner layers have $\varepsilon_{\mathrm{r}}=\varepsilon_{\mathrm{c}}$. The dielectric outside the ASR has $\varepsilon_{\mathrm{r}}=2+1.5 i$. Figure $5 \mathrm{f}$ shows the absorption spectra of the ASR metasurfaces, which can only reach $80 \%$ above $9.3 \mathrm{GHz}$ and are not comparable to that of the LSR metasurfaces in Figs. 2 and 3. This confirms the essential role of scale invariance in achieving broadband high absorption.

\section{Discussion}

We note that the performance of the LSR metasurface depends on the values of $\alpha$ and $\beta$. The constant $\alpha$ controls the size of the spiral and determines the working frequency of the metasurface. The constant $\beta$ controls how "compact" the spiral is and determines the mode density (i.e. number of resonances per frequency interval) of the LSR. When $\beta$ takes an appropriate value, the corresponding mode density results in broadband matching of the metasurface impedance with vacuum impedance and therefore significantly improves absorption. Some metamaterials constructed with fractal structures can also absorb electromagnetic waves within a broadband 
of frequencies ${ }^{36-39}$. The fractal structures are self-similar and are invariant under discrete scaling, i.e. $\chi$ takes discrete values. Self-similarity can broaden the absorption spectra of metamaterials by providing multiple resonances at discrete frequencies. But the spectral distribution of the resonances has poor tunability, leading to poor matching between the metamaterial impedance and vacuum impedance. Therefore, fractal metamaterials usually have non-smooth spectra with relatively low absorption. In contrast, the logarithmic spiral is invariant under continuous scaling transformation, i.e. $\chi$ can take arbitrary values. The scale invariance enables easy tuning of the metasurface impedance via parameter $\beta$ and the absorption spectrum is not only broadband but also smooth. There might be other geometries that are scale invariant, but the LSR has a unique property which contributes to the strong absorption of electromagnetic waves: it induces vortex flow of electromagnetic energy. The vortex channel enables efficient absorption of energy by guiding waves to travel a long way in a compact space volume. This scenario is similar to the dissipation of kinetic energy in turbulent flows in fluid dynamics, where the kinetic energy of a fluid undergoes strong damping due to the hierarchical structure of vortices: the energy is transferred from large vortices to small vortices and eventually converted to heat due to viscosity ${ }^{40}$. Here in the LSR metasurface, electromagnetic energy flows from the outer layer of the LSR (corresponding to large vortex) to the inner layers (corresponding to smaller vortices), and is eventually absorbed in the center due to material loss. The similarity between the two completely different physical systems indicates the critical role of vortex flow in the energy dissipation of classical systems.

The proposed metasurface absorber could be fabricated via the "Swiss Roll" process, which has been applied to make a prototype of the magnetic metamaterial ${ }^{41}$. This can be done by coating a plain sheet of metal (e.g. copper foil) with a wedge layer of lossy dielectric whose permittivity can be engineered to be either homogeneous or inhomogeneous. The thickness of the dielectric can be controlled to generate the designed spiral after rolling the coated metal. Then, the absorber can be produced by assembling many spirals on a large metallic screen to form a periodic structure. The proposed absorber requires dielectric materials with a relatively large imaginary part. Possible candidates are poorly conductive materials, such as conductive polymer. In addition, conductive composites can have a broad tunablity of real and imaginary parts, which can be achieved via engineering the porosity of air phase and the concentration of the conductive fillers in three-phased conductive foams.

In conclusion, we provided a general recipe for designing broadband electromagnetic wave absorbers by combining scale invariant geometry and no-cutoff TM guided mode. Following the recipe, we designed a metasurface by using LSRs which can achieve high absorption of incident microwave within a broadband of frequencies. The critical role of scale invariance is verified through a comparative study with ASR metasurface. The results may be extended to high frequency regime for the absorption of visible light. The related physics may also be applied to the absorption of other classical waves such as sound.

\section{Data Availability}

All data supporting the findings of this study are included in this published article. Additional data related to this article are available from the corresponding authors upon reasonable request.

\section{References}

1. Pendry, J. B., Holden, A. J., Robbins, D. J. \& Stewart, W. J. Magnetism from conductors and enhanced nonlinear phenomena. IEEE Trans. Microw. Theory Tech. 47, 2075-2084 (1999).

2. Smith, D. R., Padilla, W. J., Vier, D. C., Nemat-Nasser, S. C. \& Schultz, S. Composite medium with simultaneously negative permeability and permittivity. Phys. Rev. Lett. 84, 4184-4187 (2000).

3. Smith, D. R., Pendry, J. B. \& Wiltshire, M. C. K. Metamaterials and negative refractive index. Science 305, 788-792 (2004).

4. Landy, N. I., Sajuyigbe, S., Mock, J. J., Smith, D. R. \& Padilla, W. J. Perfect metamaterial absorber. Phys. Rev. Lett. 100, 207402 (2008).

5. Tao, H. et al. Highly flexible wide angle of incidence terahertz metamaterial absorber: Design, fabrication, and characterization. Phys. Rev. B 78, 241103 (2008).

6. Tao, H. et al. A metamaterial absorber for the terahertz regime: Design, fabrication and characterization. Opt. Express 16, 7181-7188 (2008).

7. Popov, E., Enoch, S. \& Bonod, N. Absorption of light by extremely shallow metallic gratings: metamaterial behavior. Opt. Express 17, 6770-6781 (2009).

8. Hao, J. et al. High performance optical absorber based on a plasmonic metamaterial. Appl. Phys. Lett. 96, 251104 (2010).

9. Liu, X., Starr, T., Starr, A. F. \& Padilla, W. J. Infrared spatial and frequency selective metamaterial with near-unity absorbance. Phys. Rev. Lett. 104, 207403 (2010).

10. Alici, K. B., Bilotti, F., Vegni, L. \& Ozbay, E. Experimental verification of metamaterial based subwavelength microwave absorbers. J. Appl. Phys. 108, 083113 (2010).

11. Zhu, B., Feng, Y., Zhao, J., Huang, C. \& Jiang, T. Switchable metamaterial reflector/absorber for different polarized electromagnetic waves. Appl. Phys. Lett. 97, 051906 (2010).

12. Cheng, Q., Cui, T. J., Jiang, W. X. \& Cai, B. G. An omnidirectional electromagnetic absorber made of metamaterials. New J. Phys. 12, $063006(2010)$.

13. Cheng, Y., Yang, H., Cheng, Z. \& Wu, N. Perfect metamaterial absorber based on a split-ring-cross resonator. Appl. Phys. A 102, 99-103 (2011).

14. Hao, J., Zhou, L. \& Qiu, M. Nearly total absorption of light and heat generation by plasmonic metamaterials. Phys. Rev. B 83, 165107 (2011).

15. Grant, J., Ma, Y., Saha, S., Khalid, A. \& Cumming, D. R. S. Polarization insensitive, broadband terahertz metamaterial absorber. Opt. Lett. 36, 3476-3478 (2011).

16. Jiang, Z. H., Yun, S., Toor, F., Werner, D. H. \& Mayer, T. S. Conformal dual-band near-perfectly absorbing mid-infrared metamaterial coating. ACS Nano 5, 4641-4647 (2011).

17. Watts, C. M., Liu, X. \& Padilla, W. J. Metamaterial electromagnetic wave absorbers. Adv. Mater. 24, OP98-OP120 (2012).

18. Wu, C. et al. Metamaterial-based integrated plasmonic absorber/emitter for solar thermo-photovoltaic systems. J. Opt. 14, 024005 (2012).

19. Dayal, G. \& Ramakrishna, S. A. Design of highly absorbing metamaterials for Infrared frequencies. Opt. Express 20, 17503-17508 (2012).

20. Liang, Q. et al. Metamaterial-based two dimensional plasmonic subwavelength structures offer the broadest waveband light harvesting. Adv. Opt. Mater. 1, 43-49 (2013). 
21. Wang, B.-Y. et al. A novel ultrathin and broadband microwave metamaterial absorber. J. Appl. Phys. 116, 094504 (2014).

22. Radi, Y., Simovski, C. R. \& Tretyakov, S. A. Thin perfect absorbers for electromagnetic waves: theory, design, and realizations. Phys. Rev. Appl. 3, 037001 (2015).

23. Lei, L., Li, S., Huang, H., Tao, K. \& Xu, P. Ultra-broadband absorber from visible to near-infrared using plasmonic metamaterial. Opt. Express 26, 5686-5693 (2018).

24. Feng, Q., Pu, M., Hu, C. \& Luo, X. Engineering the dispersion of metamaterial surface for broadband infrared absorption. Opt. Lett. 37, 2133-2135 (2012)

25. Ye, D. et al. Ultrawideband dispersion control of a metamaterial surface for perfectly-matched-layer-like absorption. Phys. Rev. Lett. 111, $187402(2013)$

26. Ding, F., Cui, Y., Ge, X., Jin, Y. \& He, S. Ultra-broadband microwave metamaterial absorber. Appl. Phys. Lett. 100, 103506 (2012).

27. Cui, Y. et al. Ultrabroadband light absorption by a sawtooth anisotropic metamaterial slab. Nano Lett. 12, 1443-1447 (2012).

28. Zhou, Z. et al. Metasurface Salisbury screen: achieving ultra-wideband microwave absorption. Opt. Express 25, 30241-30252 (2017).

29. Bilotti, F., Toscano, A., Alici, K. B., Ozbay, E. \& Vegni, L. Design of miniaturized narrowband absorbers based on resonant-magnetic Inclusions. IEEE Trans. Electromagn. Compat. 53, 63-72 (2011).

30. Zhong, S. \& He, S. Ultrathin and lightweight microwave absorbers made of mu-near-zero metamaterials. Sci. Rep. 3, 2083 (2013).

31. Huang, X. et al. Triple-band polarization-insensitive wide-angle ultra-thin planar spiral metamaterial absorber. J. Appl. Phys. 113, 213516 (2013).

32. Chen, K. et al. Microwave absorber based on permeability-near-zero metamaterial made of Swiss roll structures. J. Phys. Appl. Phys. 48, 455304 (2015).

33. Yu, D., Liu, P., Dong, Y., Zhou, D. \& Zhou, Q. A sextuple-band ultra-thin metamaterial absorber with perfect absorption. Opt. Commun. 396, 28-35 (2017).

34. Miroshnichenko, A. E. \& Tribelsky, M. I. Ultimate absorption in light scattering by a finite obstacle. Phys. Rev. Lett. 120, 033902 (2018).

35. Rozanov, K. N. Ultimate thickness to bandwidth ratio of radar absorbers. IEEE Trans. Antennas Propag. 48, 1230-1234 (2000).

36. Cheng, Y.-Z., Nie, Y. \& Gong, R.-Z. Design of a wide-band metamaterial absorber based on fractal frequency selective surface and resistive films. Phys. Scr. 88, 045703 (2013).

37. Zhu, L.-H. et al. Broadband absorption and efficiency enhancement of an ultra-thin silicon solar cell with a plasmonic fractal. Opt. Express 21, A313-A323 (2013).

38. Wu, P. C., Papasimakis, N. \& Tsai, D. P. Self-affine graphene metasurfaces for tunable broadband absorption. Phys. Rev. Appl. 6, 044019 (2016).

39. Kenney, M. et al. Octave-spanning broadband absorption of terahertz light using metasurface fractal-cross absorbers. ACS Photonics 4, 2604-2612 (2017).

40. Falkovich, G. \& Sreenivasan, K. R. Lessons from hydrodynamic turbulence. Phys. Today 59, 43-49 (2006).

41. Wiltshire, M. C. K. et al. Microstructured magnetic materials for RF flux guides in magnetic resonance imaging. Science 291, 849-851 (2001)

\section{Acknowledgements}

This work was supported by the Research Grants Council of Hong Kong SAR (No. AoE/P-02/12, No. CityU 21302018, and No. R6015-18). S. W. was also supported by grants from City University of Hong Kong (No. 7200549 and No. 9610388). B. H. was supported by the grant from Natural Science Foundation of China (NSFC) (No. 11474212) and the Priority Academic Program Development (PAPD) of Jiangsu Higher Education Institutions. We thank Prof. Z. Q. Zhang for valuable comments and suggestions.

\section{Author Contributions}

S.W. conceived the idea and performed the calculations. B.H. assisted with theoretical and numerical analysis. S.W. and C.T.C. supervised the project. All authors contributed to discussions and the writing of the manuscript.

\section{Additional Information \\ Competing Interests: The authors declare no competing interests.}

Publisher's note Springer Nature remains neutral with regard to jurisdictional claims in published maps and institutional affiliations.

Open Access This article is licensed under a Creative Commons Attribution 4.0 International License, which permits use, sharing, adaptation, distribution and reproduction in any medium or format, as long as you give appropriate credit to the original author(s) and the source, provide a link to the Creative Commons license, and indicate if changes were made. The images or other third party material in this article are included in the article's Creative Commons license, unless indicated otherwise in a credit line to the material. If material is not included in the article's Creative Commons license and your intended use is not permitted by statutory regulation or exceeds the permitted use, you will need to obtain permission directly from the copyright holder. To view a copy of this license, visit http://creativecommons.org/licenses/by/4.0/.

(C) The Author(s) 2019 